\title{
Gliding Dragons and Flying Squirrels: Diversifying versus Stabilizing Selection on Morphology following the Evolution of an Innovation
}

\author{
Terry J. Ord, ${ }^{1, \star}$ Joan Garcia-Porta, ${ }^{1, \dagger}$ Marina Querejeta, ${ }^{2, \ddagger}$ and David C. Collar ${ }^{3}$ \\ 1. Evolution and Ecology Research Centre and the School of Biological, Earth and Environmental Sciences, University of New South Wales, \\ Kensington, New South Wales 2052, Australia; 2. Institute of Evolutionary Biology (CSIC-Universitat Pompeu Fabra), Passeig Marítim \\ de la Barceloneta, 37-49, Barcelona 08003, Spain; 3. Department of Organismal and Environmental Biology, Christopher Newport \\ University, Newport News, Virginia 23606
}

Submitted August 1, 2018; Accepted July 16, 2019; Electronically published December 17, 2019

Online enhancements: supplemental material. Dryad data: https://doi.org/10.5061/dryad.t7g227h.

\begin{abstract}
Evolutionary innovations and ecological competition are factors often cited as drivers of adaptive diversification. Yet many innovations result in stabilizing rather than diversifying selection on morphology, and morphological disparity among coexisting species can reflect competitive exclusion (species sorting) rather than sympatric adaptive divergence (character displacement). We studied the innovation of gliding in dragons (Agamidae) and squirrels (Sciuridae) and its effect on subsequent body size diversification. We found that gliding either had no impact (squirrels) or resulted in strong stabilizing selection on body size (dragons). Despite this constraining effect in dragons, sympatric gliders exhibit greater size disparity compared with allopatric gliders, a pattern consistent with, although not exclusively explained by, ecological competition changing the adaptive landscape of body size evolution to induce character displacement. These results show that innovations do not necessarily instigate further differentiation among species, as is so often assumed, and suggest that competition can be a powerful force generating morphological divergence among coexisting species, even in the face of strong stabilizing selection.
\end{abstract}

Keywords: arboreality, Draco, ecological opportunity, evolutionary allometry, morphological evolution, terrestriality.

\section{Introduction}

Evolutionary innovation is any novel trait that enhances performance and allows an organism to interact with the environment in a new way (Simpson 1944). Although sev-

\footnotetext{
* Corresponding author; email: t.ord@unsw.edu.au.

$\dagger$ Present address: Department of Biology, Washington University, Saint Louis, Missouri 63130.

¥ Present address: Institut de Recherche sur la Biologie de I'Insecte, UMR 7261, CNRS, Université de Tours, Avenue Monge, Parc Grandmont 37200 Tours, France. ORCIDs: Ord, https://orcid.org/0000-0002-2608-2150; Garcia-Porta, https:// orcid.org/0000-0003-4032-9495; Collar, https://orcid.org/0000-0003-2631-6743.
}

Am. Nat. 2020. Vol. 195, pp. E51-E66. (C) 2019 by The University of Chicago. 0003-0147/2020/19502-58647\$15.00. All rights reserved.

DOI: $10.1086 / 706305$ eral definitions of what represents an innovation have been offered (reviewed by Rabosky 2017), this classical description arguably remains the most useful (Galis 2001; Stroud and Losos 2016; Rabosky 2017). Hypothesized innovations have drawn considerable attention among ecologists and evolutionary biologists because they can expand the range of ecological niches occupied within communities. In doing so, innovations are thought to be important engines of adaptive phenotypic differentiation (Price et al. 2010) and the diversification of new species (Maia et al. 2013; Silvestro et al. 2014). For example, the extensive radiation and morphological differentiation of Anolis lizards throughout the Caribbean and Americas ( 400 species; Poe 2004) has in part been attributed to the evolution of adhesive toe pads opening up an array of new arboreal environments (Losos 2009). Similar arguments have been made for the diversification of the geckos, which have also independently evolved toe pads (Garcia-Porta and Ord 2013).

In this study, we focused on the manner in which an innovation changes the evolution of phenotypic differentiation among closely related species (rather than its role in prompting species diversification). By its classical definition of facilitating the exploitation of new ecological resources, the evolution of an innovation should be especially important in promoting phenotypic adaptation to those new resources, irrespective of whether that innovation has caused species diversification or not. Yet innovations do not always herald the evolution of phenotypic diversity (Stroud and Losos 2016), and our understanding of why this is the case remains poor (Wainwright and Price 2016).

One can imagine that if the innovation enhances performance for only a limited range of morphologies, or if the increase in performance comes at a cost to other performance capabilities, ecological specialization might result, 
and stabilizing selection for performance could limit phenotypic diversification (Wainwright and Price 2016; Alencar et al. 2017). While toe pad evolution has been informally linked to morphological diversification in lizards (e.g., Warheit et al. 1999), recent investigations of the geckos have found little evidence of such an association (GarciaPorta and Ord 2013). In contrast, gecko lineages that have lost their toe pads exhibit elevated morphological evolution, which suggests that toe pads might have actually imposed a functional constraint on phenotypic diversification (e.g., reduced locomotor performance; Higham et al. 2015). An innovation might therefore open up a new ecological niche (e.g., one associated with being arboreal), but the breadth of that niche could be narrow and restricted to a specialized morphology (e.g., a gracile or small body).

The notion that innovations can restrain, rather than promote, phenotypic differentiation is largely unexplored, but it has broad implications for our general understanding of when adaptive evolution is likely to occur via other processes as well. In particular, ecological competition among interacting members (intra- or interspecific) can drive adaptive morphological differentiation through character displacement, a phenomenon that seems especially important in adaptive radiations (e.g., Grant and Grant 2006; Stuart et al. 2014; Lescak et al. 2015). Here, taxa diverge in resource use to reduce competition with coexisting congeners, which prompts changes in morphology (e.g.) as taxa adapt to their changed resource base. This raises the general question of how ecological competition interacts with innovations to generate phenotypic diversity. Innovations are generally assumed to prompt adaptive differentiation, but competition could provide the ecological context that promotes the origin of the innovation or act as a complementary factor to facilitate further phenotypic differentiation via character displacement (Givnish 1997; Stroud and Losos 2016). But an innovation that promotes phenotypic diversification in the absence of competition could reduce the opportunity for character displacement to arise in the first place.

For character displacement to occur, ecologically similar sympatric species must initially coexist before competition can drive selection that ultimately pushes species to diverge in resource use and subsequent functional morphology. But the potential for this initial coexistence is questionable. Hutchinson (1959) highlighted how species must differ in ecology in order to coexist in the same environment. That is, there is a necessary limiting similarity among sympatric species (May and MacArthur 1972; Schoener 1974); otherwise species will be excluded from areas in which an ecologically similar competitor already exists (e.g., Colautti et al. 2006). This filtering or species sorting subsequently results in species communities that were already differentiated in resource use and morphology at the outset (NB: there are situations in which characteristics related to competitive ability - e.g., accelerated development, large body sizecould result in ecologically similar species existing in a highcompetition environment; e.g., see Mayfield and Levine 2010).

How these processes play out may depend on whether an innovation promotes or constrains phenotypic and ecological diversity. In the case of an innovation that drives ecological and phenotypic diversification, there is a greater chance that species have phenotypically differentiated before occurring in sympatry. That is, there are fewer opportunities for ecologically similar species to co-occur-and fewer opportunities for character displacement-simply because there are fewer ecologically similar species following the origin of an innovation. On the other hand, if an innovation imposes stabilizing selection, competition might override innovation-imposed constraints to prompt character displacement to alternative adaptive optima (assuming ecologically similar species are able to coexist long enough for divergent selection to occur).

Gliding is a striking example of an innovation but one that is ambiguous in its performance-based constraints on morphology. Controlled aerial descent in the form of gliding or parachuting has evolved independently in various arthropod, reptilian, amphibian, and mammalian groups (reviewed by Dudley et al. 2007) and has resulted in a unique way for animals to exploit arboreal environments. Gliding has also been suggested to be an adaptation for escaping predation (Byrnes and Spence 2011; McGuire and Dudley 2011), which should reduce any predator-mediated restrictions on the range of ecological resources that might be available to gliding taxa (see Stroud and Losos 2016). Gliding has therefore been considered an innovation that has lead to an array of ecological opportunities or release from predation, and, in doing so, has the potential to promote further adaptive differentiation in morphology.

Yet gliding imposes important physical constraints on morphology, especially as it relates to gross body size. Larger animals are expected to be poorer gliders compared with smaller animals because of their increased weight (e.g., Losos et al. 1989; McGuire 2003), which reduces the distance over which a larger glider can travel (McGuire and Dudley 2005). This constraint should result in selection that effectively caps the size of gliding species. But it could also be possible that larger gliders circumvent this constraint by evolving disproportionately large wing membranes to compensate for their increased weight (e.g., Shine et al. 1998). The outcome might instead be little to no performance constraint on body size evolution, allowing gliders to morphologically diversify as they transition into a range of new arboreal niches.

We investigated the evolutionary history of gliding, its performance in relation to body size, and its subsequent 
effects on body size evolution in two iconic gliding groups: flying squirrels from the family Sciuridae and gliding dragons from the family Agamidae. If the evolution of gliding provided new ecological opportunities or an ecological release from predation, squirrel and dragon species should have diversified substantially in size as they colonized new, previously unavailable, or underexploited arboreal niches. On the other hand, if gliding imposes constraints on body size, we expected species to exhibit reduced size disparity associated with a history of stabilizing selection.

In the particular case of gliding dragons (genus Draco), prior work by McGuire and others has set strong expectations regarding the interplay between intrinsic constraints and ecological competition. Larger species do not seem to compensate for their increased weight by having larger wing membranes (Colbert 1967; McGuire 2003; but see Shine et al. 1998) and are generally poorer gliders than smaller species as an apparent consequence (McGuire and Dudley 2005). Although the reduction in gliding performance with size should constrain body size evolution, gliding dragons seem to vary widely in size (Colbert 1967; McGuire 2003), prompting speculation that this disparity might reflect character displacement (McGuire 2003). There is, in fact, some evidence of ecological competition among sympatric species (Inger 1983) that appears to have led to habitat partitioning (Ord and Klomp 2014) and body size disparities that might be consistent with character displacement (Husak and McGuire 2014). Although these patterns are suggestive of roles for innovation-imposed constraints and competition-driven divergence, no study has evaluated how these factors have interacted to shape size evolution throughout the clade or the extent to which morphological disparity among sympatric species is an outcome of species sorting rather than character displacement. Gliding dragons therefore provide a means of investigating in a natural system the balance of potentially opposing selection pressures on size (Shine et al. 1998): gliding performance-driven selection for small size (Shine et al. 1998; McGuire 2003) versus competition-driven selection that pushes some lizards to larger sizes (Inger 1983; Husak and McGuire 2014; Ord and Klomp 2014) or excludes them from sympatric communities entirely.

Flying squirrels offer an intriguing comparison with dragons. Like Draco, they possess wing membranes that extend along the trunk and control aerial movement between trees. However, the underlying anatomy of the wing differs between squirrels (Thorington and Heaney 1981) and dragons (Colbert 1967), and the precise scaling of the wing surface and its consequences for gliding performance in squirrels are unknown (see also "Methods"). Squirrels therefore provide an opportunity to evaluate the generality of the diversification consequences of gliding. However, squirrels were less amenable for examining the interaction between the innovation and competition because little evidence of competition is reported in this group, and widespread sympatry among flying squirrels (all except one species included in our study were sympatric with other flying squirrels) effectively precluded a proper test of character displacement. Nonetheless, the anatomical differences of the squirrel gliding membrane compared with that of gliding dragons provides a means of potentially identifying the specific performance constraints that might be imposed on size.

\section{Methods \\ Overview}

To investigate how gliding has affected body size evolution, we combined species data on body size and lifestyle (whether they were gliders, arboreal, or terrestrial) from the literature and our own observations in the field. These data were then coupled with new phylogenies developed from molecular data obtained from GenBank in order to apply statistical methods designed to reveal the potential dynamics of phenotypic evolution.

We focused on body size in our study of morphological differentiation for two reasons. First, body size (and mass in particular) is an important determinant of gliding performance (Losos et al. 1989; McGuire 2003; McGuire and Dudley 2005). Any conflict in selection pressures associated with ecological diversification and competition versus those maintaining gliding performance should be especially clear in the dynamics of body size evolution. Second, and more generally, body size is correlated to a host of other phenotypic, metabolic, and ecological characteristics and provides a general index for investigating adaptive phenotypic diversification among taxa occupying different environmental niches and habitats (e.g., Clauset and Erwin 2008; Collar et al. 2011; Okie et al. 2013; Feldman et al. 2016). Divergence in body size is also a common outcome of character displacement among sympatric species (e.g., Schluter and McPhail 1992; Pfennig and Murphy 2003; Kirschel et al. 2009).

We applied a range of evolutionary analyses to explore various aspects of the origin of gliding and how it has altered size evolution in dragons and squirrels. First, we explored the origins of gliding through ancestor state reconstructions to gain a general view of the evolutionary history of gliding and its relationship with other lifestyles (e.g., Has gliding evolved from an arboreal lifestyle? Has gliding evolved once or several times within each family?). Second, we assessed whether the origin of gliding led to changes in the evolutionary relationship between body mass and length through phylogenetic regressions and the extent to which changes in body size have affected gliding performance more specifically. Third, we estimated the 
rates of adaptation and stochastic evolution in body size along lineages that were gliders relative to other lifestyles (arboreality and terrestriality). Finally, for gliding dragons, we examined the possibility that species interacting with congeners exhibit body size divergences consistent with character displacement, using evolutionary null models of body size evolution and a deterministic model of ecological competing species.

\section{Data}

Body Size, Lifestyle, and Gliding Performance. We used two data sets of body size for the dragon lizards (both originally compiled from a variety of sources by Feldman et al. [2016], including original data on Draco reported in McGuire [1998]; we also supplemented these data with an additional species from Kalahroudi and Hojati [2015]). The first data set included maximum snout-to-vent length (SVL; $n=458$ species; table S1; tables S1-S14 are available online), while the second covered a more limited set of species but included data on mean SVL ( $\mathrm{mm})$ and mean mass (g; $n=110$ species; table S2). For the squirrels, we compiled data on species means for length ( $n=220$ species $)$ and mass (g; $n=210$ species) from a variety of sources (table S3). Information on lifestyle (arboreal, terrestrial, and gliding) for both families ( $n=295$ dragon species, $n=$ 183 squirrel species) was obtained from a variety of sources (tables S4, S5). To clarify the probable effect of body size on gliding performance, we used data on wing load from two sources (gliding dragons, Draco, McGuire 1998; flying squirrels of various genera, Thorington and Heaney 1981).

Distributions of Gliding Dragons (and Flying Squirrels). Categorization of Draco species as occurring in allopatry (13 species) or sympatry (21 species; NB: sympatric communities ranged from pairs of species to as many as eight at a single site - see table S6) was based on the personal observations of the first author (T.J.O.) as well as several comprehensive field guides (Grismer 2011) and biogeographic studies reporting detailed information on site distributions (Musters 1983; McGuire and Alcala 2000; McGuire and Heang 2001; McGuire et al. 2007). Species in allopatry were those found on single-species islands, while species in sympatry were those found on islands, large landmasses (e.g., Borneo, Sulawesi), or the mainland where at least one other Draco species had been observed or reported to co-occur at the same site. That is, our categorization of sympatry was intended to reflect a high probability of those species encountering at least one congener. Efforts were also made to find comparable data for flying squirrels, but this proved problematic for the reasons outlined in the introduction and supplemental material (available online).

\section{Phylogeny}

We developed new phylogenies for the dragon lizards and squirrels using molecular data retrieved from GenBank (Benson et al. 2012; tables S7, S8). Methodological details are provided in full in the supplemental material. A concise summary is given below.

For the dragons, we used five genes that previous studies have shown provide the greatest coverage of species (Pyron et al. 2013). Our final alignment of these genes included 297 species (including two outgroups) and a total of 4,637 bp distributed as follows: 12S rRNA (12S, 1,103 bp), 16S rRNA (16S, 582 bp), NADH dehydrogenase subunit 2 (ND2, $951 \mathrm{bp}$ ), brain-derived neurotrophic factor (BDNF, 690 bp), and RAG1 (1,311 bp). For the squirrels, we used six genes frequently used by previous phylogenetic studies of this family (Mercer and Roth 2003; Fabre et al. 2012) and included 220 species and a total of 6,001 bp distributed as follows: $12 \mathrm{~S}$ rRNA (12S, $378 \mathrm{bp}), 16 \mathrm{~S}$ rRNA (16S, $367 \mathrm{bp}$ ), cytochrome oxydase 3 (COX3, $783 \mathrm{bp}$ ), cytochrome b (CYTB, 1,140 bp), interphotoreceptor retinoid-binding protein (IRBP, 1,188 bp), and recombination-activating protein 1 (RAG1, 2,145 bp).

Phylogenetic analyses were conducted using the package BEAST 1.8.0 (Drummond and Rambaud 2007) under a lognormal relaxed molecular clock (Drummond and Rambaud 2007) and two fossil calibration points per family. For both families, three independent Markov chain Monte Carlo (MCMC) chains were run for 50,000,000 generations each and sampled every 5,000. Tracer version 1.6 (Rambaut and Drummond 2007) was used to confirm convergence, good mixing, and stationarity of each MCMC chain. After excluding $10 \%-30 \%$ of trees as burn-in (dependent on the number generations for each chain to reach a stationary distribution), the posterior sets of trees sampled in each run were combined using LogCombiner 1.8.0 (available in the BEAST package). We then used TreeAnnotator 1.8.0 (available in the BEAST package) to calculate summary trees for each family (figs. S2, S3; figs. S1-S9 are available online). These represented the maximum clade credibility trees with a posterior probability limit of 0.5 . We also reserved a sample of 1,000 posterior trees to assess the sensitivity of subsequent phenotypic analyses to phylogenetic uncertainty. These latter analyses are presented in full in the supplemental material and were consistent with findings obtained from summary trees.

\section{Phenotypic Analyses}

All analyses were implemented in R version 3.4.0 ( $\mathrm{R}$ Development Core Team, R Foundation for Statistical Computing, Vienna). Body size and wing load were natural log transformed prior to analyses. Details on statistical methods 
not outlined below, including those associated with the sample of 1,000 posterior trees, are provided in the supplemental material.

Ancestral State Reconstructions. We used stochastically mapped ancestor state reconstructions (Huelsenbeck et al. 2003) to obtain a general view of the evolutionary history of lifestyle within each family. These reconstructions also provided the foundation for testing differences in morphological evolution among lifestyles (see "Estimated Rates of Adaptation and Stochastic Evolution" below). Reconstructions were computed using phytools 0.6-00 (Revell 2012) and consisted of 1,000 alternative reconstructions estimated along the summary tree for each family. These were used to visualize the history of lifestyle evolution, as well as for subsequent analysis of morphological evolution as a function of lifestyle.

Evolutionary Regressions. We used phylogenetically informed regressions to test the hypothesis that the origin of gliding led to a shift in the evolutionary relationship between body mass and body length. We applied several regression models that considered a variety of alternative scenarios in which the intercepts and slopes of mass on length were assumed to be different or the same among lifestyles. The level of support for each model was then compared using the corrected Akaike information criterion $\left(\mathrm{AIC}_{\mathrm{c}}\right)$, with any model within two units of the lowest estimated value considered to be equally credible $\left(\Delta \mathrm{AIC}_{\mathrm{c}} \leq 2.0\right)$. The best-supported model $\left(\Delta \mathrm{AIC}_{\mathrm{c}}=0.0\right)$ was also used to compute residuals of mass to consider mass-specific evolutionary change free of the influence of body length.

Given our a priori expectation that body size has evolved under strong selection imposed by lifestyle (especially gliding), we applied regressions using models that assumed an Ornstein-Uhlenbeck (OU) process implemented in the slouch package version 1.2 (Hansen et al. 2008). This method uses maximum likelihood to estimate the rate of adaptation pulling the phenotype toward an optimum and deviations from the optimum caused by unconsidered factors. These estimates are presented as phylogenetic half-life $\left(t_{1 / 2}\right)$, which is the length of time in millions of years that size has taken to evolve halfway from its ancestral state to its inferred adaptive optimum and the magnitude of the stationary variance $\left(v_{y}\right)$ of species around the optimum. These parameters were also converted to their integrals to allow comparison with other methods reporting the estimated rate of adaptation $(\alpha)$ and the extent to which random fluctuations $\left(\sigma^{2}\right)$ have occurred in the process of size evolution (e.g., see "Estimated Rates of Adaptation and Stochastic Evolution"). When $t_{1 / 2}$ is very large or $\alpha$ converges on zero, the model collapses to a purely Brownian motion (BM) process (Hansen et al. 2008).
To investigate the extent to which evolutionary increases in body size have led to loss of gliding performance, we reevaluated the evolutionary allometry of wing load for dragons and formally investigated it for the first time in flying squirrels. An allometric analysis of wing load in Draco species has previously been conducted by McGuire (2003; using the data from McGuire 1998; but see also Colbert 1967 and Shine et al. 1998) and the relationship was reported to be isometric. Our goal was to clarify this relationship using allometric analyses that implemented a more biologically relevant evolutionary process (an OU model) and one based on updated phylogenetic information. In the case of the squirrels, an allometric estimate of gliding performance has yet to be conducted, although Thorington and Heaney (1981) provide an informal discussion of wing load as a function of body size, and some have interpreted this discussion to imply that gliding performance is isometric (McGuire 2003).

To estimate allometry of wing load, we applied the same approach described above that involved phylogenetic regression under an OU process (Hansen et al. 2008). We regressed the natural logarithm of wing load on the natural logarithm of body length, $L$, according to the equation: $\ln ($ wingload $)=a+\ln (L) b$. If larger species have compensated for their increased weight through the evolution of disproportionately larger gliding membranes, wing load (body mass divided by wing area) should exhibit a negative allometric scaling relationship with body length (i.e., slope coefficient $<1.0$ ). If, on the other hand, wing load scales isometrically with body length (i.e., slope coefficient = 1.0 ), there has been no compensation for weight through the evolution of increased wing area. In this case, larger species are expected to suffer a steeper reduction in gliding performance.

Estimated Rates of Adaptation and Stochastic Evolution. We tested for lifestyle-specific patterns of adaptive evolution in two measures of body size-body length (species mean SVL in dragons and its equivalent in squirrels) and length-standardized mass (i.e., species residual mass from evolutionary regression against length, referred to hereafter as residual mass). We began by applying three alternative models of how each size variable might have evolved as a function of lifestyle: (i) a BM-only model in which lifestyle has had no effect on size evolution; (ii) an OU model in which size evolution has differed between arboreal and nonarboreal lineages (with gliders grouped with other arboreal species); and (iii) an OU model in which size evolution has differed between arboreal, terrestrial, and gliding lifestyles. These models were implemented using OUwie 1.50 (Beaulieu et al. 2012) and in the case of the two OU models were based on the 1,000 stochastic reconstructions of lifestyle (arboreal or nonarboreal; arboreal, terrestrial, or 
glider; Huelsenbeck et al. 2003) mapped onto the summary tree of each family. Error in stochastic mapping on subsequent estimates of evolutionary rates in a secondary continuous variable (e.g., body size) has been shown to reduce statistical power and the ability to distinguish differences in evolutionary rate of the secondary variable among stochastically mapped categories (Revell 2013). In this sense, any difference found in the evolutionary dynamics among reconstructed lifestyle categories is likely to reflect a large biological effect, whereas the lack of difference could be type II error if a lifestyle varies widely across the phylogeny.

In all cases, the OU model in which the evolution of body size (length or residual mass) has been free to vary among arboreal, terrestrial, and gliding lineages was found to be the most probable scenario (based on a comparison of $\mathrm{AIC}_{\mathrm{c}}$, although the magnitude of support for this model varied depending on the variable and family examined; see "Results"). We therefore focused our attention on this model to interpret lifestyle-specific $\alpha$ and $\sigma^{2}$ for all runs that estimated the optimum value of body size, $\theta$, to be within a biologically realistic range - those that occurred between the minimum measured empirical value $-1 \mathrm{SD}$ and the maximum measured empirical value $+1 \mathrm{SD}$ (following Price et al. 2015). We also explored the effect of potential error in measures of body size on our parameter estimates and found that outcomes were virtually unchanged compared with those presented in "Results" (details of how within-species variance was compiled and used in OUwie analyses, as well as the results of those analyses, are provided in full in the supplemental material).

Tests of Character Displacement in Gliding Dragons. To test the hypothesis that competition among sympatric Draco species has promoted body size divergence (McGuire 2003), we calculated differences in body length and residual mass among co-occurring congeners and compared these values with null distributions of differences generated by simulating evolution without regard to species co-occurrence. We obtained 1,000 simulated data sets performed along the Draco phylogeny under both BM and OU processes in which parameters were allowed to vary across the mid-95\% range obtained from OUwie-estimated values. All simulations were performed using phytools (Revell 2012). For each simulated data set, we estimated the mean Euclidean distance of body size among sympatric species and the mean Euclidean distance of body size among allopatric species. Because character displacement is predicted to produce greater morphological distances among sympatric species relative to allopatric species, these distances were subtracted from one another to obtain null distributions of apparent character displacement. This method allowed us to determine whether sympatric species were in fact more dissimilar than would be expected given an undirected (nonadaptive) evo- lutionary process (i.e., stochasticity via $\mathrm{BM}$ or $\mathrm{OU}$ ) and the length of time size differentiation has had to occur. It is important to note that we used this test to evaluate whether the disparity pattern was consistent with character displacement, but it does not exclude other possible evolutionary processes, such as species sorting (i.e., competitive exclusion resulting in only dissimilar congeners being able to coexist) or adaptive divergence prompted by other factors.

We also used Clarke et al.'s (2017) model of ecological competing species as a second test of the character displacement hypothesis. While this more deterministic model similarly cannot distinguish between character displacement and some other process, it goes some way in testing the extent to which disparity has accrued in a manner that is consistent with competition among sympatric species occurring over evolutionary time. However, this test relied on assumptions that are likely ill suited to the biological context of our study system (outlined in the supplemental material). We consequently focused our investigation on the outcome of empirical comparisons to the null simulations. We note that results from Clarke's model were consistent and are described in full in the supplemental material.

We applied the model using the treecomp package (modified ver. 2016 from Github; Clarke et al. 2017), which simulates body size evolution along the phylogeny of Draco using a theoretical model of competition applied under a BM process. The method applies the simulation under two scenarios. The first (BM only) assumed that ecological competition is absent and that body size has evolved purely by a BM process. The second scenario (BM plus competition) assumed that ecological competition has occurred among sympatric species, such that the body size of one sympatric species has influenced the body size evolution of another sympatric species. We then tested the likelihood of obtaining the empirically observed distribution of body length and mass residuals across the tips of the phylogeny against these simulated scenarios.

\section{Results \\ Evolutionary History of Lifestyle Shifts}

Both families appear to have originated from arboreal ancestors, with a variety of subsequent transitions to a terrestrial lifestyle. The evolution of gliding occurred only once in both families: at 25-35 Ma in squirrels and 20-23 Ma in dragon lizards (fig. 1).

\section{Length-Weight Relationships}

In the agamid lizards, the best-supported model indicated a prominent divergence in mass between gliders and nongliders (table S9, pt. a). Specifically, gliding dragons were 
(a) Dragons

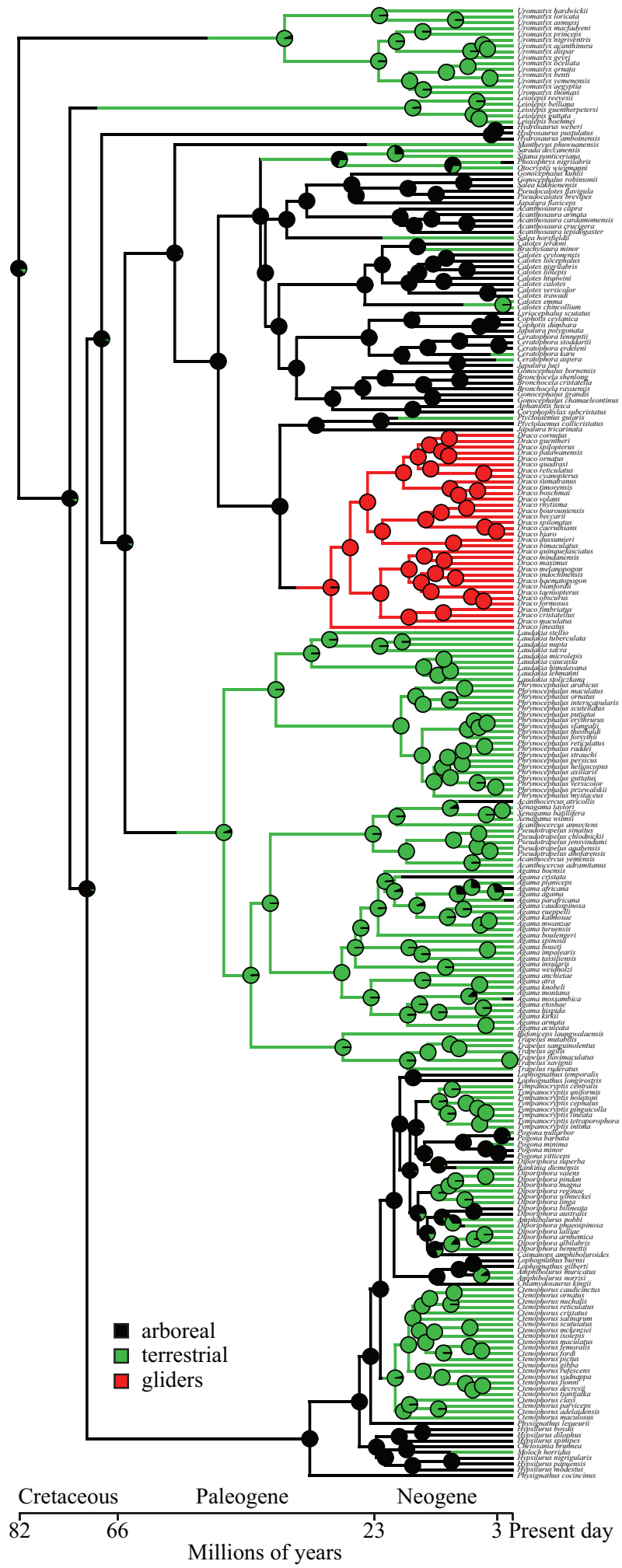

(b) Squirrels (and marmots)

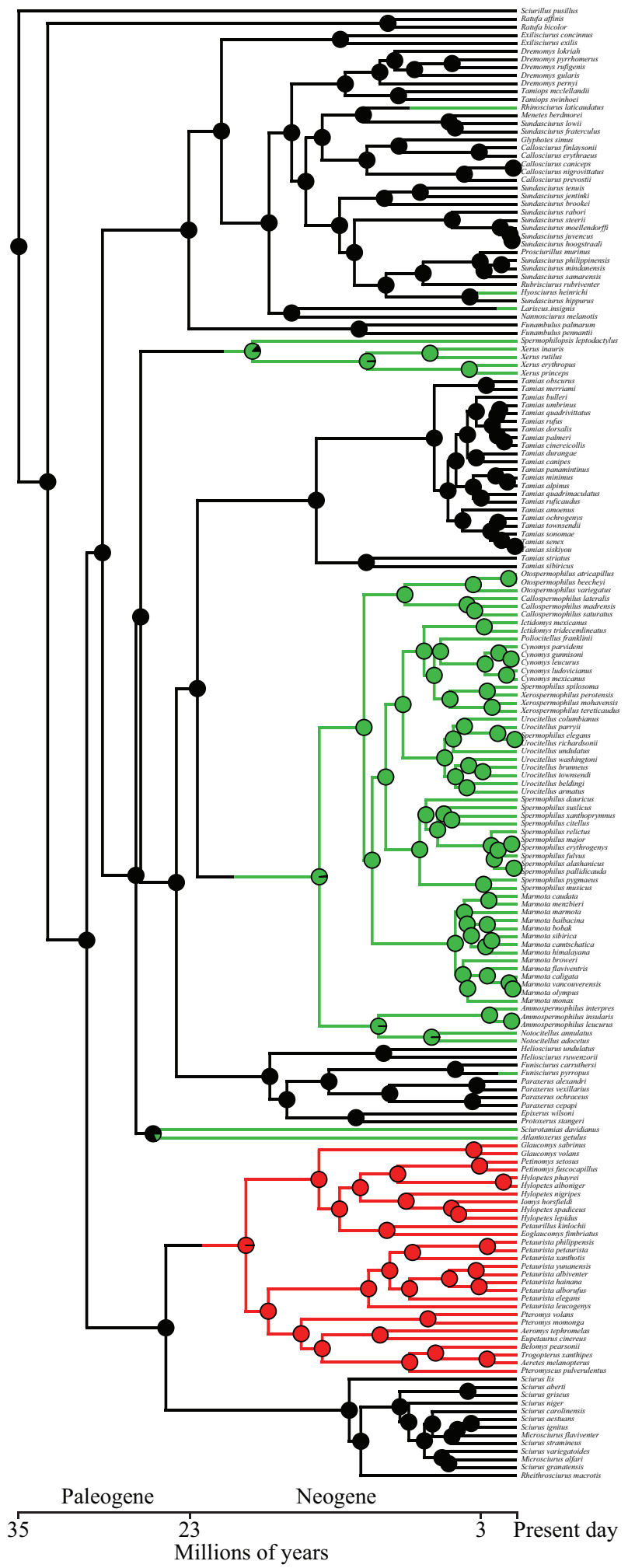

Figure 1: Evolutionary history of arboreal, terrestrial, and gliding dragon lizards ( $a$; Agamidae) and squirrels ( $b$; Sciuridae). Pie charts at phylogenetic nodes illustrate the proportion of 1,000 stochastically mapped reconstructions assigned to a given lifestyle. Color assignments on phylogenetic branches illustrate a single representative reconstruction. 
considerably lighter for a given body length compared with both arboreal and terrestrial lizards (fig. $2 a$ ).

In squirrels, the null model in which all lifestyles followed the same scaling relationship of mass on body length was the most credible model (table S9, pt. b; fig. 2b). An alternative model in which mass differed in gliders and nongliders also received support (table S9, pt. b), but the ef-

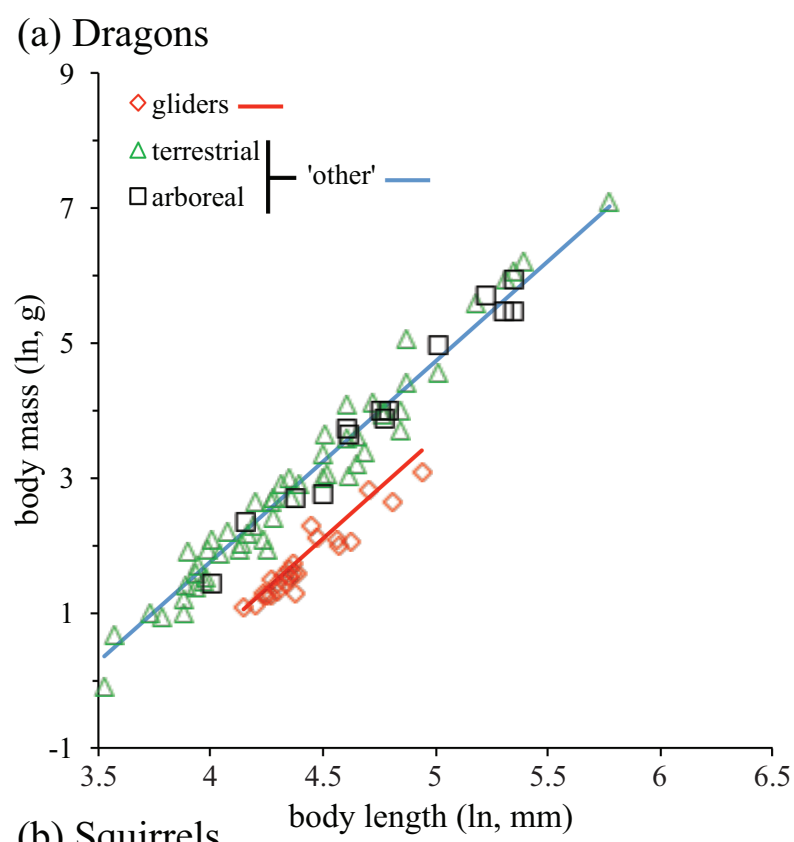

(b) Squirrels

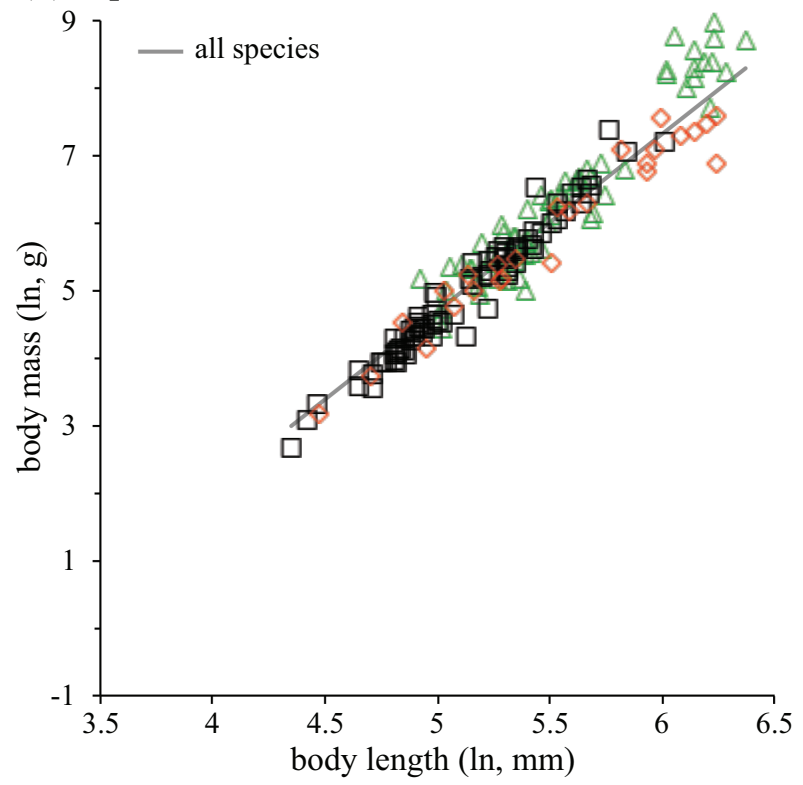

Figure 2: Relation between body mass and length in arboreal, terrestrial, and gliding dragon lizards $(a)$ and squirrels $(b)$. Trend lines depicted in main plots are evolutionary regressions computed from family summary trees (reported in table S11). fect size for this variable in the model was not significantly different from zero and the model was not considered further (table S11, pt. b).

\section{Body Size Evolution}

In the agamid lizards, the OU model in which the evolution of body length has been free to vary among arboreal, terrestrial, and gliding lineages was the best supported of the three alternative models applied (figs. $3 a$, S4, upper panels). Parameter estimates from this model showed that the rate of adaptation $(\alpha)$ of body length appears to have been substantially higher in gliders than in arboreal and terrestrial lineages, with a very low rate of stochastic evolution $\left(\sigma^{2}\right.$; fig. 3i). This implies a history of intense stabilizing selection, which has tightly constrained gliding dragons to an inferred adaptive optimum $(\theta)$ of smaller sizes relative to arboreal and terrestrial lineages (fig. $3 i$ ). In addition, there was little evidence for any change in body length evolution among nongliding arboreal or terrestrial lineages.

Although gliding lizards exhibited reduced mass for their body length compared with nongliders, we found little evidence that body mass at any given length has been more tightly constrained in this group. In other words, despite differences in mass-length regressions between gliders and nongliders (fig. 2a), residual mass exhibited similar patterns of evolution in these groups (fig. S4i). In fact, there was little empirical support for differences in residual mass evolution among lifestyle groups, in general. Although some analyses inferred that nongliding arboreal lizard lineages may have experienced strong stabilizing selection (high $\alpha$, low $\sigma^{2}$ ) on residual mass, suggesting constraints on variation in mass for a given body length, this result depended on the ancestral lifestyle reconstruction applied (fig. S4i; see also fig. S5ii).

In squirrels, although the OU model in which the evolution of size has been free to vary among arboreal, terrestrial, and gliding lineages was generally the best-supported model, for body length in particular this support was low relative to the alternative evolutionary models applied (figs. $3 b, \mathrm{~S} 4 b$, upper panels). Inspection of the lifestyle-specific parameter estimates from this model showed that while arboreal lineages have tended to evolve toward shorter body lengths (a lower inferred adaptive optima, $\theta$ ) relative to terrestrial and gliding squirrels (fig. $3 b$; see also fig. S5iii), there were no other consistent differences in length or mass evolution among lifestyles (any difference in estimates of $\alpha$ and $\sigma^{2}$ were dependent on the ancestral reconstruction considered; figs. $3 b, \mathrm{~S} 4 b)$.

\section{Wing Load Allometry}

Gliding dragons exhibited isometric scaling as previously reported (McGuire 2003): the slope of the evolutionary 
(a) Dragons

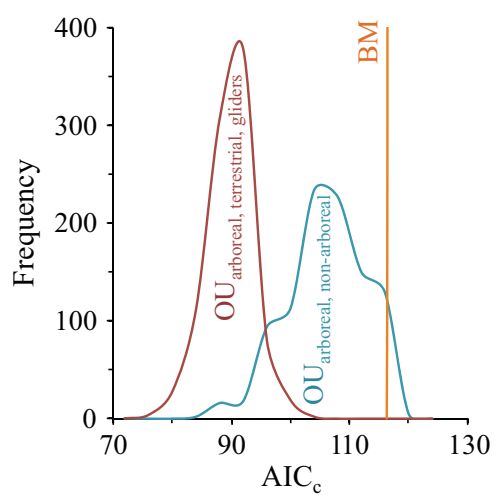

(i) $\mathrm{OU}_{\text {arboreal, terrestrial, gliders }}$
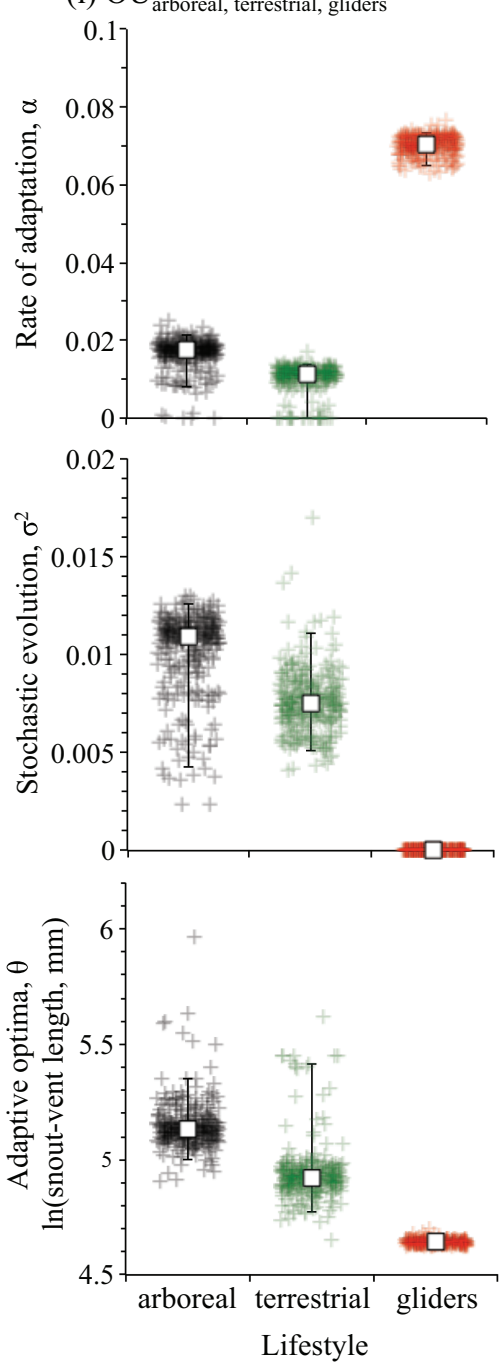

(b) Squirrels
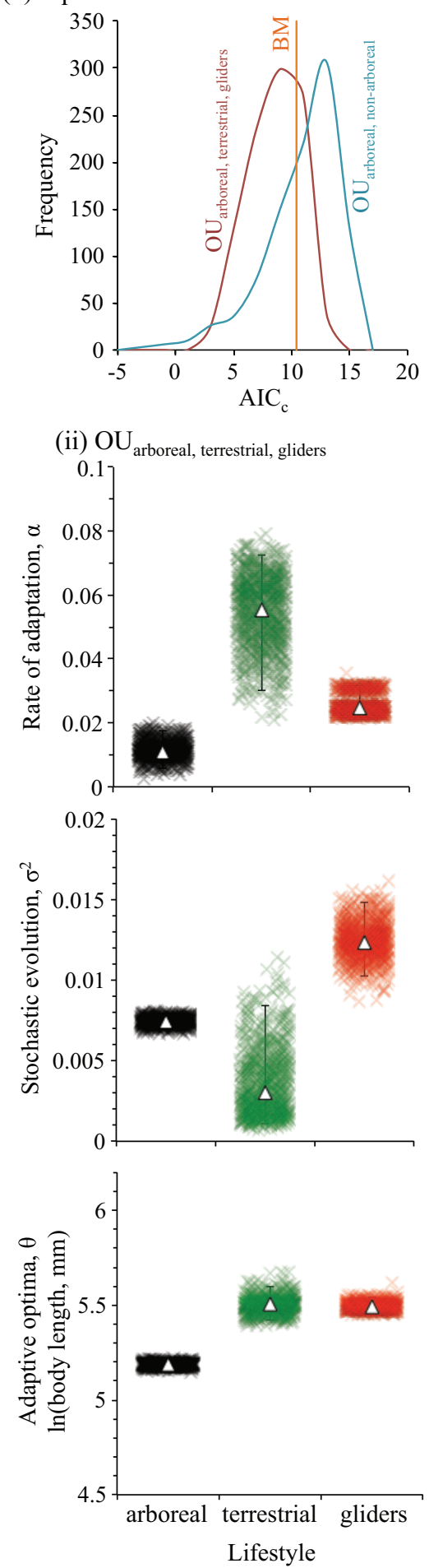

Figure 3: Body length evolution in dragon lizards $(a)$ and squirrels $(b)$. Depicted in the uppermost panels are the levels of support for three alternative models of size evolution. Illustrated in the lower panels $(i, i i)$ are the parameter estimates derived from the Ornstein-Uhlenbeck $(\mathrm{OU})$ model in which size evolution has been allowed to vary among arboreal, terrestrial, and gliding species. Parameters reflect the inferred rate of adaptation $(\alpha)$ in body length, its rate of stochastic evolution $\left(\sigma^{2}\right)$, and its inferred adaptive optima $(\theta)$. Colored data points are the individual estimates computed from each of the 1,000 separate stochastically mapped reconstructions of lifestyle. These data are summarized as a median and 95th percentile range overlay. $\mathrm{AIC}_{\mathrm{c}}=$ corrected Akaike information criterion; $\mathrm{BM}=$ Brownian motion. 
Table 1: Evolutionary relationships between wing load $\left(\mathrm{N} / \mathrm{m}^{2}\right)$ and body length $(\mathrm{mm})$ in gliding dragon lizards (Agamidae) and squirrels (Sciuridae)

\begin{tabular}{lllll}
\hline & $\beta_{0}(95 \% \mathrm{CI})$ & $\beta_{\mathrm{InL}}(95 \% \mathrm{CI})$ & $\alpha$ (support region) & $\sigma^{2}$ (support region) \\
\hline $\begin{array}{l}\text { Gliding dragon lizards } \\
\left.\quad n_{\text {species }}=27\right)\end{array}$ & $-.97(-2.15$ to .21$)$ & $.81(.55$ to 1.08$)$ & $.001(0, .002)$ & $.05(.19, \infty)$ \\
Squirrels $\left(n_{\text {species }}=11\right)$ & $.69(.66$ to .72$)$ & $.63(.626$ to .638$)$ & $.03(.0, \infty)$ & $.00(0, .08)$ \\
\hline
\end{tabular}

Note: We report the estimated rate of adaptation $(\alpha)$ and the extent to which random fluctuations $\left(\sigma^{2}\right)$ have occurred in the process of body mass evolution. These parameters are given with a confidence range of values within two likelihood units of the best-supported value. CI $=$ confidence interval.

regression of wing load on body length was not significantly different from 1 (table 1; fig. 4). That is, there was no evidence that larger gliding dragons have compensated for their increased size by developing larger wings to reduce wing load. Because wing load is inversely proportional to the loss of altitude over a given horizontal distance (McGuire and Dudley 2005), isometric evolution of wing load suggests that larger gliding dragons are poorer gliders. We also determined that allopatric and sympatric species conformed to the same allometric model of wing load (i.e., sympatric species did not differ in intercept or slope from allopatric species in their allometry; see table S12).

In contrast, flying squirrels exhibited negative allometric scaling, contradicting previous assumptions about this relationship (Thorington and Heaney 1981; McGuire 2003): the slope of the evolutionary regression of wing load on body length was significantly lower than 1 (table 1; fig. 4). This indicates that larger flying squirrels have in part compensated for their increased size by increasing the area of their wings to reduce wing load. Despite this, gliding dragons exhibited substantially lower wing loads than squirrels, suggesting that dragons are better gliders overall compared with similar-sized flying squirrels (fig. 4).

\section{Divergences among Sympatric Gliding Dragons}

Sympatric Draco species exhibited greater variance in body length and residual mass compared to species occurring in allopatry (fig. 5) and this was unlikely to have occurred under either a BM or a single-optimum OU process (body length: BM $P<.001$, OU $P<.001$; residual mass: BM $P=.01$, OU $P<.001)$. This pattern of putative competition-driven divergence between sympatric species was generally confirmed by findings from Clarke et al.'s (2017) model (table S14).

\section{Discussion}

Evolutionary innovations that open up new ecological opportunities and competition that pushes congeners to diverge in resource use and subsequent morphology have both been described as important drivers of phenotypic di- versification among closely related taxa (Givnish 1997; Galis 2001; Pfennig and Pfennig 2012; Stroud and Losos 2016). For example, one or both factors have been implicated in the origin of exceptional ecomorphological differentiation in several iconic adaptive radiations (e.g., Anolis, Stuart et al. 2014; three-spine sticklebacks, Lescak et al. 2015; and Darwin's finches, Grant and Grant 2006). As a general paradigm for explaining adaptive diversification in nature, debate has largely centered on how often evolutionary innovations might arise (Vermeij 2006; Erwin 2015; Wagner 2017; see also Blount et al. 2008; Meyer et al. 2012) and the extent to which competition promotes outcomes such as character displacement rather than the exclusion

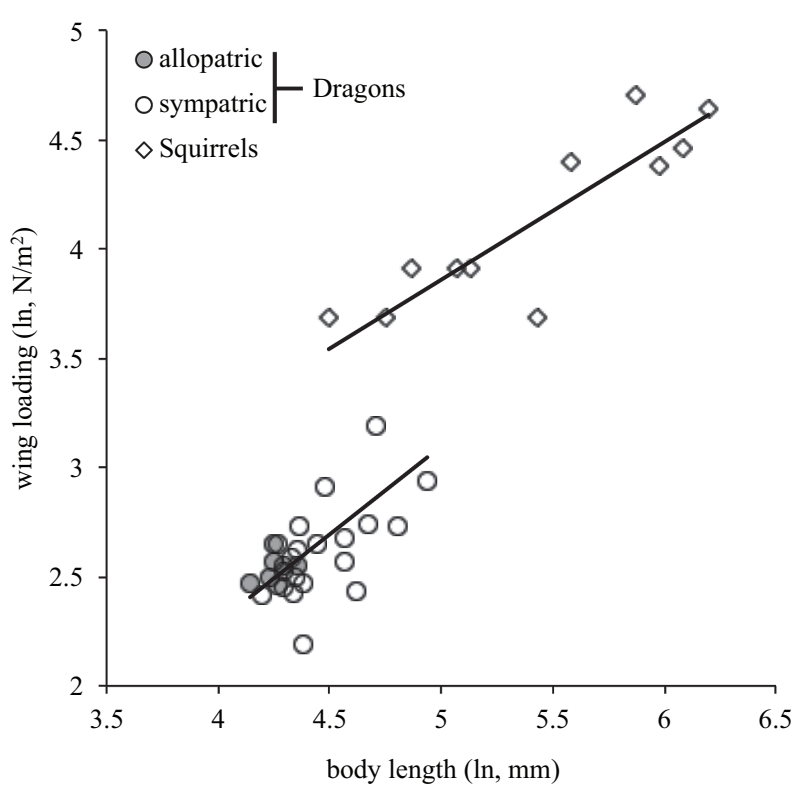

Figure 4: Evolutionary relationships between wing load and body length in gliding dragon lizards and squirrels. Wing load was reported by sources as $\mathrm{N} / \mathrm{m}^{2}$ (Thorington and Heaney 1981; McGuire 1998), with $\mathrm{N}$ being mass in newtons calculated as mass multiplied by a constant reflecting acceleration due to gravity and $\mathrm{m}^{2}$ being the surface area of the gliding membrane. Higher wing load values correspond to lower gliding performance (e.g., shorter gliding distances and greater losses of altitude while gliding; McGuire and Dudley 2005). Trend lines depicted in main plots are evolutionary regressions computed using Hansen's et al. (2008) Ornstein-Uhlenbeck process. 
(a) Body length

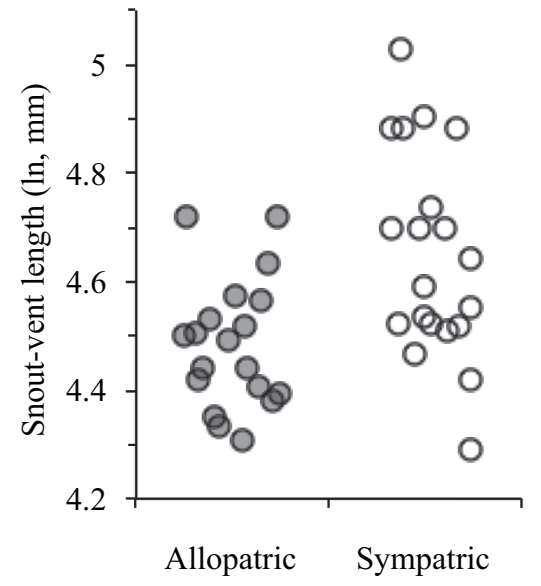

(b) Body mass

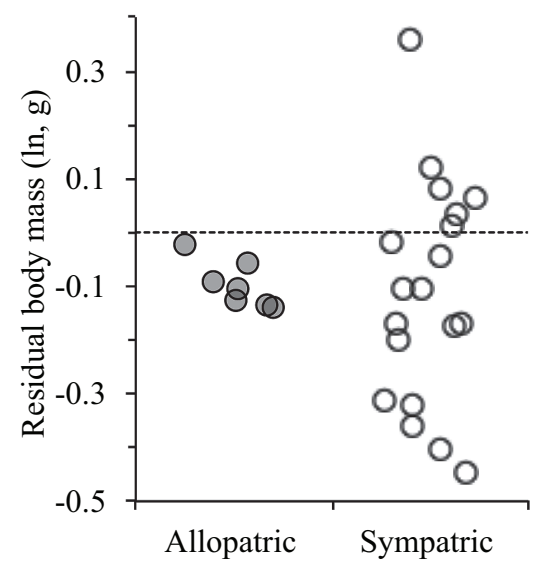

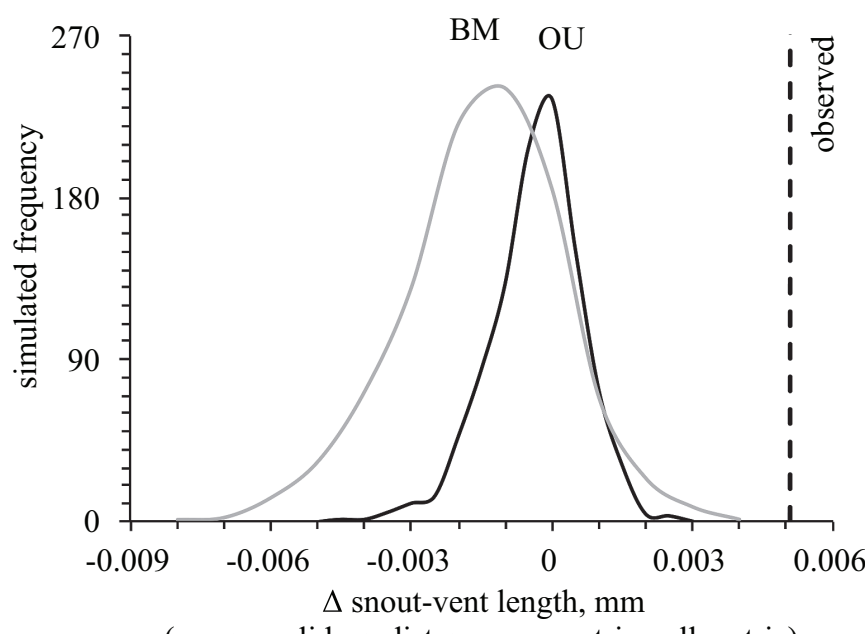

(mean euclidean distance, sympatric - allopatric)

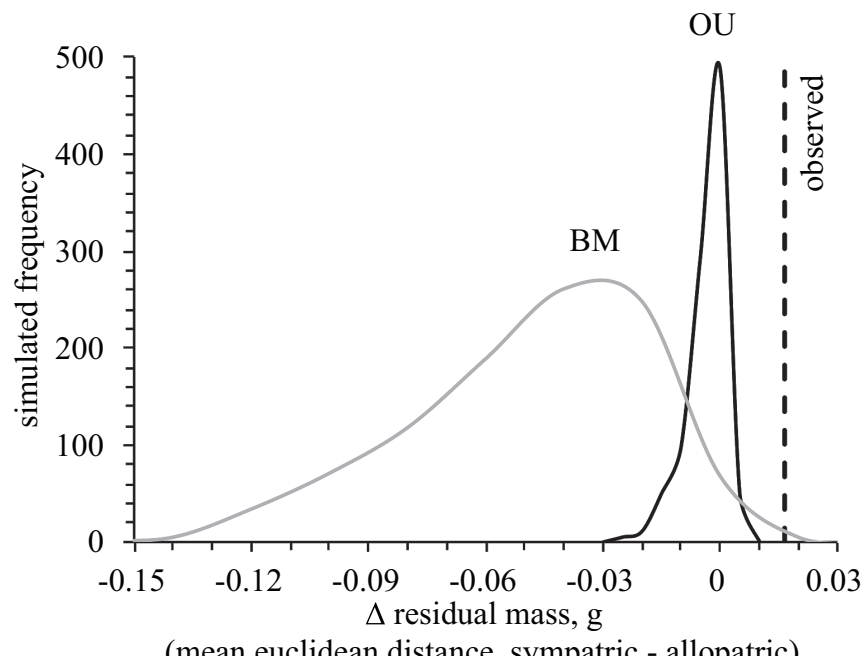

Figure 5: Gliding Draco dragon lizards exhibit pronounced body length $(a)$ and mass $(b)$ differentiation in sympatry relative to allopatry that is unlikely to have arisen by chance. Plots on the left illustrate the observed empirical data, while plots on the right show null distributions of body size evolution as the difference in mean Euclidean distance between sympatric and allopatric species simulated under Brownian motion (BM) or an Ornstein-Uhlenbeck (OU) process. The dashed line illustrates the observed mean Euclidean distance between sympatric and allopatric species computed from empirical data.

of ecologically similar species from coexisting more generally (Dayan and Simberloff 2005; Emerson and Gillespie 2008; Rabosky 2013; Stuart and Losos 2013). However, there is also the additional question of whether innovations, when they evolve, actually spur diversification (Stroud and Losos 2016; Wainwright and Price 2016). The general expectation has been that they do (Hunter 1998; Schluter 2000; Coyne and Orr 2004; Vermeij 2006; Kelley and Pyension 2015), but that outcome is far from guaranteed (Wainwright and Price 2016).
As an innovation, the evolution of gliding is a classic one that has occurred repeatedly across several extant (Losos et al. 1989; Dudley et al. 2007; Heinicke et al. 2012) and extinct lineages (McGuire and Dudley 2011; Brusatte 2017; Han et al. 2017). Its origin in living animals is believed to have been an adaptation to improve the cost of transport in an arboreal environment (Dial 2003), which in turn should have allowed gliding animals to more effectively compete or exploit resources (e.g., food, territories; Byrnes and Spence 2011; McGuire and Dudley 2011). It may also have allowed 
gliding animals to more effectively avoid predators (Byrnes and Spence 2011; McGuire and Dudley 2011). Our analysis is the first to quantitatively confirm the arboreal origins of gliding in both groups, which has been the focus of much speculation (Colbert 1967; Thorington and Heaney 1981; Losos et al 1989). The evolution of an arboreal lifestyle as a prerequisite for gliding is intuitive and probably widespread (Dudley et al. 2007; Meng et al. 2017; NB: this is unlikely to be the case for the evolution of active flight, the origins of which remain controversial although often linked to a terrestrial lifestyle [Dudley et al. 2007; Brusatte 2017]). The general timing of the evolution of gliding in both families (35-20 Ma) was also roughly comparable to the inferred spread of highcanopied rain forests throughout Southeast Asia (50-20 Ma), which has been implicated in the evolution of gliding in geckos (Heinicke et al. 2012). Regardless of the adaptive reasons for its initial evolution, the transition from an arboreal to a gliding lifestyle (fig. 1) is expected to have resulted in new ecological opportunities and a release from predation for both flying squirrels and gliding dragons (Dudley et al. 2007).

The role of ecological competition in adaptive phenotypic differentiation is also unclear, although (like innovations) it is widely believed to be instrumental (Pfennig and Pfennig 2012; Rabosky 2013; Stroud and Losos 2016). There are many putative examples of competition leading to character displacement (e.g., Dayan and Simberloff 2005), but many of these might also be explained by other phenomena (Stuart and Losos 2013). For example, while comparison between allopatric and sympatric populations of the same species is particularly powerful for revealing character displacement, differences among populations can still arise because of divergent selection that is unrelated to competition per se (e.g., differences in other biotic or abiotic conditions between sympatric and allopatric populations). Such considerations are especially problematic when comparing allopatric versus sympatric species because species are expected to differ in a number of unconsidered ecological dimensions. It is also difficult to exclude the possibility that species already differed in ecology and morphology before coexistence (which would also be consistent with competitive exclusion resulting in species sorting). However, these confounding factors are potentially dampened if species experience some type of stabilizing pressure that has tended to keep both allopatric and sympatric species tracking toward the same optimal phenotype. In sympatry, some species are pushed off this primary optima because of the secondary factor of competition, but in the absence of competition allopatric species are able to converge on the same optimal phenotype (sensu Simpson 1944; see also Hansen 1997).

In the case of dragons, our study has shown that gliding has placed critical limits on body size evolution and that these limits should be experienced by both allopatric and sympatric species. In fact, it seems that other aspects of Draco morphology (head, body, and limb dimensions) are evolutionarily constrained in this way as well (see Collar et al. 2010). We found no evidence that larger gliding dragons have compensated for their increased size by evolving disproportionately larger wings, which is also consistent with previous findings (McGuire 2003; but see Shine et al. 1998). Bigger dragons have therefore almost certainly experienced the increased cost associated with being poorer gliders compared with smaller dragons. The patagium in gliding dragons is supported across elongated thoracic ribs that in turn control the extension and retraction of the wing (Colbert 1967). The length of these ribs, and subsequently the size of the wing, are potentially restricted by the distance between the forearm and hind leg (McGuire and Dudley 2005). Although increasing the length of the body would increase rib length, the potential benefit to gliding performance would be offset by the consequent increase in body mass.

Our study shows that Draco have evidently responded to these demands by evolving a more gracile body form: compared with other agamids of similar length, Draco have reduced mass, which provides for more favorable wing loading than they would exhibit had they maintained the masslength relationship of other agamids. Nevertheless, Draco are still limited to relatively small sizes (figs. 2, 4) because wing load scales isometrically among species (McGuire 2003; this study); larger species have not increased wing area and/or reduced their body weight any more than what has occurred for the genus as a whole. Moreover, the increased size disparity seen among sympatric Draco is less than that observed in nongliding agamid groups of similar evolutionary age - for example, body size ranges of sympatric Draco were 6\%-26\% less than Agama, 50\%-61\% less than Ctenophorus, and 23\%-72\% less than Phrynocephalus - which is again consistent with the notion of stabilizing selection constraining Draco body size evolution (and in sympatry where character displacement in body size might have occurred).

Yet despite strong stabilizing selection on small body sizes for most gliding dragons, those living in sympatry exhibited elevated size disparity compared with allopatric species. This phenomenon had been noted previously (McGuire 2003; Husak and McGuire 2014), but our study offers quantitative confirmation. While we argue that this pattern supports widespread character displacement, it does not exclude the possibility that competitive exclusion resulted in species sorting. Because of the apparent size limits imposed by gliding performance, however, the plausibility of this alternative explanation rests on the identification of selective factors that could overcome this constraint to drive size divergence in allopatry.

In the case of our data, additional insight might also come from the fact that allopatric Draco species were usually 
from islands, whereas many of the sympatric communities in our analyses were from larger landmasses. This is potentially important because many animals often exhibit a tendency toward smaller body sizes on islands compared with mainland environments (and vice versa: the so-called island rule; e.g., Lomolino 2005). It is possible then that consistently small body sizes observed in allopatric Draco reflects something similar, while the disparity seen in sympatric mainland communities reflects species sorting following earlier divergence in a more heterogeneous environment in allopatry, although the exact circumstances leading to this divergence are still unknown.

Although our results do not exclude a possible role for species sorting, we favor the explanation that the disparity difference in sympatry versus allopatry arose because of character displacement. According to this scenario, resource competition among co-occurring species would have contributed to divergent selection strong enough to push species away from a gliding performance-imposed size optimum. In fact, evidence suggests that sympatric populations of Draco diverge in resource use from allopatric populations of the same species, and the resulting partitioning of the environment in sympatry has occurred in a manner consistent with resource competition (in some cases, sympatric species are found at different perch heights of the same tree; Inger 1983; Ord and Klomp 2014). Furthermore, such partitioning has also been linked to divergence in other morphology as well (e.g., limb length; Ord and Klomp 2014), which in itself might be evolutionarily constrained by gliding (see Collar et al. 2010). If character displacement has occurred, our results indicate that competition is capable of inducing adaptive size differentiation among sympatric species despite limits imposed by strong selection opposing increases in body size. If size disparity among sympatric species has not been instigated by competition, it still remains unclear why these species have diverged in body size given the concomitant loss in gliding performance.

Selection trade-offs are likely to be common in nature, and strong stabilizing selection that limits the ability of taxa to efficiently diverge in resource use would generally be expected to result in competitive exclusion (e.g., McGee et al. 2015). In fact, it seems remarkable that competition among sympatric species has resulted in character displacement and not competitive exclusion; assuming the average body size of Draco species in allopatry is close to optimal for gliding, the largest sympatric species - that is, those in the upper quartile - probably suffer a $26 \%-55 \%$ reduction in gliding performance (based on the allometric equations in table 1, pt. A, and data from McGuire and Dudley 2005). It is possible that larger Draco species have compensated for their poorer gliding ability in other ways. For example, our field observations suggest reduced gliding in heavier species (fig. S9a) and a shift to more cryptic antipredator behavior (fig. S9b), presumably because it offsets less effective gliding as a means of escape for larger species. Yet it is clear that gliding is central to the behavioral ecology of all Draco and has greatly expanded the arboreal niche for these lizards. Its origin and maintenance also implies that gliding conveys important fitness benefits. That is, any behavioral changes that might evolve to reduce the effect of poor gliding performance in large species are likely a case of making the best of a bad job. These considerations are relevant regardless of what might have prompted shifts to larger body sizes in sympatry.

Evolutionary patterns in flying squirrels contrast strongly with those inferred for dragons. Squirrels were poorer gliders than dragons overall (fig. 4), but the negative allometric scaling of wing load in squirrels (table 1) does indicate that larger species have been able to develop disproportionately larger patagia, which has helped reduce the effect of weight on gliding performance (to a degree). Unlike Draco, the patagium in flying squirrels attaches to the fore- and hind limbs, so wing area can potentially increase with the evolution of longer limbs. Presumably, positive evolutionary allometry in limb length has allowed wing area to increase at a faster rate than mass, and this in turn seems to have enabled squirrels to escape the strong stabilizing selection that is so apparent for gliding dragons (the rates of adaptation and stochastic evolution in body size of flying squirrels was largely no different than those of nongliding species; fig. $3 b$ ). At the same time, our result provides no evidence that gliding has spurred diversification of body size among gliding squirrels.

There are a growing number of other cases in which the evolution of an innovation seems to have failed to promote significant phenotypic evolution (see Garcia-Porta and Ord 2013 and Wainwright and Price 2016), and in the case of gliding dragons, we show that it can instead result in a severe constraint on morphological change. In addition, gliding dragons might offer an important illustration of just how powerful ecological competition might be in driving phenotypic differentiation among coexisting species. Competition has seemingly not only driven a wedge between many of these species (sensu Pfennig and Pfennig 2012) but potentially pushed several off an adaptive peak that has been critical for the evolutionary success of the genus as a whole. However, it is notoriously difficult to document ecological character displacement definitively in nature (reviewed by Stuart and Losos 2013), and future investigation of gliding dragons in this regard is certainly warranted. If gliding dragons ultimately prove to be anything to go by, competition could be a critical engine of adaptive diversification capable of offsetting performance-based constraints from functionally specialized innovations and other opposing selection pressures. 


\section{Acknowledgments}

This study was partly supported by an Endeavour Research Fellowship to J.G.P. awarded by the Department of Education and Training, Australian Government (ID: 5471_ 2016). We thank Isaac Casanovas for his advice about squirrel fossils. Pioneering studies by Dick Thorington, Larry Heaney, and Jim McGuire provided key foundations for this work and inspired our own investigation into the potentially competing evolutionary challenges faced by gliding animals.

\section{Data and Code Availability}

All data have been deposited in the Dryad Digital Repository (https://doi.org/10.5061/dryad.t7g227h; Ord et al. 2020).

\section{Literature Cited}

Alencar, L. R. V. d., M. Martins, G. Burin, and T. B. Quental. 2017. Arboreality constrains morphological evolution but not species diversification in vipers. Proceedings of the Royal Society B 284: 20171775.

Beaulieu, J. M., D.-C. Jhwueng, C. Boettiger, and B. C. O'Meara. 2012. Modeling stabilizing selection: expanding the Ornstein-Uhlenbeck model of adaptive evolution. Evolution 66:2369-2383.

Benson, D. A., M. Cavanaugh, K. Clark, I. Karsch-Mizrachi, D. J. Lipman, J. Ostell, and E. W. Sayers. 2012. GenBank. Nucleic Acids Research 41:D36-D42.

Blount, Z. D., C. Z. Borland, and R. E. Lenski. 2008. Historical contingency and the evolution of a key innovation in an experimental population of Escherichia coli. Proceedings of the National Academy of Sciences of the USA 105:7899-7906.

Brusatte, S. L. 2017. A Mesozoic aviary: biomechanical models are key to understanding how dinosaurs experimented with different ways of flying. Science 355:792-794.

Byrnes, G., and A. J. Spence. 2011. Ecological and biomechanical insights into the evolution of gliding in mammals. Integrative and Comparative Biology 51:991-1001.

Clarke, M., G. H. Thomas, and R. P. Freckelton. 2017. Trait evolution in adaptive radiations: modeling and measuring interspecific competition on phylogenies. American Naturalist 189:121-137.

Clauset, A., and D. H. Erwin. 2008. The evolution and distribution of species body size. Science 321:399-401.

Colautti, R. I., I. A. Grigorovich, and H. J. MacIsaac. 2006. Propagule pressure: a null model for biological invasions. Biological Invasions 8:1023-1037.

Colbert, E. H. 1967. Adaptations for gliding in the lizard Draco. American Museum Novitates 2283:1-20.

Collar, D. C., J. A. Schulte II, B. C. O’Meara, and J. B. Losos. 2010. Habitat use affects morphological diversification in dragon lizards. Journal of Evolutionary Biology 23:1033-1049.

Collar, D. C., J. A. Schulte II, and J. B. Losos. 2011. Evolution of extreme body size disparity in monitor lizards (Varanus). Evolution 65:2664-2680.

Coyne, J. A., and H. A. Orr. 2004, Speciation. Sinauer, Sunderland, MA.

Dayan, T., and D. Simberloff. 2005. Ecological and community-wide character displacement: the next generation. Ecology Letters 8: 875-894.
Dial, R. 2003. Energetic savings and the body size distributions of gliding mammals. Evolutionary Ecology Research 5:1151-1162.

Drummond, A. J., and A. Rambaut. 2007. BEAST: Bayesian evolutionary analysis by sampling trees. BMC Evolutionary Biology 7:214.

Dudley, R., G. Byrnes, S. P. Yanoviak, B. Borrell, R. M. Brown, and J. A. McGuire. 2007. Gliding and the functional origins of flight: biomechanical novelty or necessity? Annual Review of Ecology, Evolution, and Systematics 38:179-201.

Emerson, B. C., and R. G. Gillespie. 2008. Phylogenetic analysis of community assembly and structure over space and time. Trends in Ecology and Evolution 23:619-630.

Erwin, D. H. 2015. Novelty and innovation in the history of life. Current Biology 25:R930-R940.

Fabre, P.-H., L. Hautier, D. Dimitrov, and E. J. Douzery. 2012. A glimpse on the pattern of rodent diversification: a phylogenetic approach. BMC Evolutionary Biology 12:88.

Feldman, A., N. Sabath, R. A. Pyron, I. Mayrose, and S. Meiri. 2016. Body sizes and diversification rates of lizards, snakes, amphisbaenians and the tuatara. Global Ecology and Biogeography 25:187-197.

Galis, F. 2001. Key innovations and radiations. Pages 581-605 in G. P. Wagner, ed. The character concept in evolutionary biology. Academic, San Diego, CA.

Garcia-Porta, J., and T. J. Ord. 2013. Key innovations and island colonization as engines of evolutionary diversification: a comparative test with the Australasian diplodactyloid gekkotans. Journal of Evolutionary Biology 26:2662-2680.

Givnish, T. J. 1997. Adaptive radiations and molecular systematics: issues and approaches. Pages 1-54 in T. J. Givnish, and K. J. Systma, eds. Molecular evolution and adaptive radiation. Cambridge University Press, Cambridge.

Grant, P. R., and B. R. Grant. 2006. Evolution of character displacement in Darwin's finches. Science 313:224-226.

Grismer, L. L. 2011. Lizards of peninsular Malaysia, Singapore and their adjacent archipelagos. Chimaira, Frankfurt.

Han, G., F. Mao, S. Bi, Y. Wang, and J. Meng. 2017. A Jurassic gliding euharamiyidan mammal with an ear of five auditory bones. Nature 551:451-455.

Hansen, T. F. 1997. Stabilizing selection and the comparative analysis of adaptation. Evolution 51:1341-1351.

Hansen, T. F., J. Pienaar, and S. H. Orzack. 2008. A comparative method for studying adaptation to a randomly evolving environment. Evolution 62:1965-1977.

Heinicke, M. P., E. Greenbaum, T. R. Jackman, and A. M. Bauer. 2012. Evolution of gliding in Southeast Asian geckos and other vertebrates is temporally congruent with dipterocarp forest development. Biology Letters 23:994-997.

Higham, T. E., A. V. Birn-Jeffery, C. E. Collins, C. D. Hulsey, and A. P. Russell. 2015. Adaptive simplification and the evolution of gecko locomotion: morphological and biomechanical consequences of losing adhesion. Proceedings of the National Academy of Sciences of the USA 112:809-814.

Huelsenbeck, J. P., R. Nielsen, and J. P. Bollback. 2003. Stochastic mapping of morphological characters. Systematic Biology 52:131-158.

Hunter, J. P. 1998. Key innovations and the ecology of macroevolution. Trends in Ecology and Evolution 13:31-36.

Husak, J. F., and J. A. McGuire. 2014. Does "gliding while gravid" explain Rensch's rule in flying lizards? Biological Journal of the Linnean Society 113:270-282.

Hutchinson, G. E. 1959. Homage to Santa Rosalia or why are there so many kinds of animals? American Naturalist 93:145-159. 
Inger, R. F. 1983. Morphological and ecological variation in the flying lizards (genus Draco). Fieldiana Zoology 18:1-33.

Kalahroudi, M., and V. Hojati. 2015. The female reproductive cycle of the spotted toad-headed agama, Phrynocephalus maculatus (Sauria: Agamidae) in Iran. Iranian Journal of Animal Biosystematics 10: 185-194.

Kelley, N. P., and N. D. Pyenson. 2015. Evolutionary innovation and ecology in marine tetrapods from the Triassic to the Anthropocene. Science 348:301.

Kirschel, A. N. G., D. T. Blumstein, and T. B. Smith. 2009. Character displacement of song and morphology in African tinkerbirds. Proceedings of the National Academy of Sciences of the USA 19: 8256-8261.

Lescak, E. A., S. L. Bassham, J. Catchen, O. Gelmond, M. L. Sherbick, F. A. von Hippel, and W. A. Cresko. 2015. Evolution of stickleback in 50 years on earthquake-uplifted islands. Proceedings of the National Academy of Sciences of the USA 112:E7204-E7212.

Lomolino, M. V. 2005. Body size evolution in insular vertebrates: generality of the island rule. Journal of Biogeography 32:1683-1699.

Losos, J. B. 2009. Lizards in an evolutionary tree: ecology and adaptive radiation of anoles. University of California Press, Berkeley.

Losos, J. B., T. J. Papenfuss, and J. R. Macey. 1989. Correlates of sprinting, jumping and parachuting performance in the butterfly lizard, Leiolepis belliani. Journal of Zoology 217:559-568.

Maia, R., D. R. Rubenstein, and M. D. Shawkey. 2013. Key ornamental innovations facilitate diversification in an avian radiation. Proceedings of the National Academy of Sciences of the USA 110: 10687-10692.

May, R. M., and R. H. MacArthur. 1972. Niche overlap as a function of environmental variability. Proceedings of the National Academy of Sciences of the USA 69:1109-1113.

Mayfield, M. M., and J. M. Levine. 2010. Opposing effects of competitive exclusion on the phylogenetic structure of communities. Ecology Letters 13:1085-1093.

McGee, M. D., S. R. Borstein, R. Y. Neches, H. H. Buescher, O. Seehausen, and P. C. Wainwright. 2015. A pharyngeal jaw evolutionary innovation facilitated extinction in Lake Victoria cichlids. Science 350:1077-1079.

McGuire, J. A. 1998. Phylogenetic systematics, scaling relationships, and the evolution of gliding performance in flying lizards (genus Draco). $\mathrm{PhD}$ diss. University of Texas at Austin.

- 2003. Allometric prediction of locomotor performance: an example from southeast Asian flying lizards. American Naturalist 161:337-349.

McGuire, J. A., and A. C. Alcala. 2000. A taxonomic revision of the flying lizards (Iguania: Agamidae: Draco) of the Philippine Islands, with a description of a new species. Herpetological Monographs 14:81-138.

McGuire, J. A., and R. Dudley. 2005. The cost of living large: comparative gliding performance in flying lizards (Agamidae: Draco). American Naturalist 166:93-106.

- 2011. The biology of gliding in flying lizards (genus Draco) and their fossil and extant analogs. Integrative and Comparative Biology 51:983-990.

McGuire, J. A., and K. B. Heang. 2001. Phylogenetic systematics of southeast Asian flying lizards (Iguania: Agamidae: Draco) as inferred from mitochondrial DNA sequence data. Biological Journal of the Linnean Society 72:203-229.

McGuire, J. A., R. M. Brown, Mumpuni, A. Riyanto, and N. Andayani. 2007. The flying lizards of the Draco lineatus group (Squamata:
Iguania: Agamidae): a taxonomic revision with descriptions of two new species. Herpetological Monographs 21:179-212.

Meng, Q.-J., D. M. Grossnickle, D. Liu, Y.-G. Zhang, A. I. Neander, Q. Ji, and Z.-X. Luo. 2017. New gliding mammaliaforms from the Jurassic. Nature 548:291-296.

Mercer, J. M., and V. L. Roth. 2003. The effects of Cenozoic global change on squirrel phylogeny. Science 299:1568-1572.

Meyer, J. R., D. T. Dobias, J. S. Weitz, J. E. Barrick, R. T. Quick, and R. E. Lenski. 2012. Repeatability and contingency in the evolution of a key innovation in Phage Lambda. Science 335:428-432.

Musters, C. J. M. 1983. Taxonomy of the genus Draco L. (Agamidae, Lacertilia, Reptilia). Zoologische Verhandelingen 199:1-120.

Okie, J. G., A. G. Boyer, J. H. Brown, D. P. Costa, S. K. M. Ernest, A. R. Evans, M. Fortelius, et al. 2013. Effects of allometry, productivity and lifestyle on rates and limits of body size evolution. Proceedings of the Royal Society B 280:20131007.

Ord, T. J., and D. A. Klomp. 2014. Habitat partitioning and morphological differentiation: the Southeast Asian Draco lizards and the Caribbean Anolis lizards compared. Oecologia 175:651-666.

Ord, T. J., J. Garcia-Porta, M. Querejeta, and D. C. Collar. 2020. Data from Gliding dragons and flying squirrels: diversifying versus stabilizing selection on morphology following the evolution of an innovation. American Naturalist, Dryad Digital Repository, https:// doi.org/10.5061/dryad.t7g227h.

Pfennig, D. W., and P. J. Murphy. 2003. A test of alternative hypotheses for character divergence between coexisting species. Ecology 84:1288-1297.

Pfennig, D. W., and K. S. Pfennig. 2012. Evolution's wedge: competition and the origins of diversity. University of California Press, Berkeley.

Poe, S. 2004. Phylogeny of anoles. Herpetological Monographs 18:3789.

Price, S. A., P. C. Wainwright, D. R. Bellwood, E. Kazancioglu, D. C. Collar, and T. J. Near. 2010. Functional innovations and morphological diversification in parrotfishes. Evolution 64:3057-3068.

Price, S. A., S. T. Freidman, and P. C. Wainwright. 2015. How predation shaped fish: the impact of fin spines on body form evolution across teleosts. Proceedings of the Royal Society B 282:20151428.

Pyron, R. A., F. T. Burbrink, and J. J. Wiens. 2013. A phylogeny and revised classification of Squamata, including 4161 species of lizards and snakes. BMC Evolutionary Biology 13:93.

Rabosky, D. L. 2013. Diversity-dependence, ecological speciation, and the role of competition in macroevolution. Annual Review of Ecology, Evolution, and Systematics 44:481-502.

Rabosky, D. L. 2017. Phylogenetic tests for evolutionary innovation: the problematic link between key innovations and exceptional diversification. Philosophical Transactions of the Royal Society B 372:20160417.

Rambaut, A., and A. J. Drummond. 2007. Tracer version 1.4. http:// beast.bio.ed.ac.uk/Tracer.

Revell, L. J. 2012. phytools: an R package for phylogenetic comparative biology (and other things). Methods in Ecology and Evolution 3:217-223.

- 2013. A comment on the use of stochastic character maps to estimate evolutionary rate variation in a continuously valued trait. Systematic Biology 62:339-345.

Schluter, D. 2000. The ecology of adaptive radiation. Oxford University Press, New York.

Schluter, D., and J. D. McPhail. 1992. Ecological character displacement and speciation in sticklebacks. American Naturalist 140:85-108. 
Schoener, T. W. 1974. Resource partitioning in ecological communities. Science 185:27-39.

Shine, R., S. Keogh, P. Doughty, and H. Giragossyan. 1998. Costs of reproduction and the evolution of sexual dimorphism in a "flying lizard" Draco melanopogon (Agamidae). Journal of Zoology (London) 246:203-213.

Silvestro, D., G. Zizka, and K. Schulte. 2014. Distantangling the effects of key innovations on the diversification of Bromelioideae (Bromeliaceae). Evolution 68:163-175.

Simpson, G. G. 1944. Tempo and mode in evolution. Columbia University Press, New York.

Stroud, J. T., and J. B. Losos. 2016. Ecological opportunity and adaptive radiation. Annual Review of Ecology, Evolution, and Systematics 47:507-532.

Stuart, Y. E., and J. B. Losos. 2013. Ecological character displacement: glass half full or half empty? Trends in Ecology and Evolution 28:402-408.

Stuart, Y. E., T. S. Campbell, P. A. Hohenlohe, R. G. Reynolds, L. J. Revell, and J. B. Losos. 2014. Rapid evolution of a native species following invasion by a congener. Science 346:463-466.

Thorington, R. W., and L. R. Heaney. 1981. Body proportions and gliding adaptations of flying squirrels (Petauristinae). Journal of Mammalogy 62:101-114.

Vermeij, G. J. 2006. Historical contingency and the purported uniqueness of evolutionary innovations. Proceedings of the National Academy of Sciences of the USA 103:1804-1809.

Wagner, A. 2017. The white-knight hypothesis, or does the environment limit innovations? Trends in Ecology and Evolution 32:131140.

Wainwright, P. C., and S. A. Price. 2016. The impact of organismal innovation on functional and ecological diversification. Integrative and Comparative Biology 56:479-488.

Warheit, K. I., J. D. Forman, J. B. Losos, and D. B. Miles. 1999. Morphological diversification and adaptive radiation: a comparison of two diverse lizard clades. Evolution 53:1226-1234.

\section{References Cited Only in the Online Enhancements}

Abascal, F., R. Zardoya, and M. J. Telford. 2010. TranslatorX: multiple alignment of nucleotide sequences guided by amino acid translations. Nucleic Acids Research 38:W7-W13.

Alvarez-Sierra, M.A., R. Daams, and P. Pelaez-Campomanes. 1999. The late Oligocene rodent faunas of Canales (MP28) and Parrales (MP29) from the Loranca Basin, Province of Cuenca, Spain. Revista Española de Paleontología 14:93-116.

Barbera, X., L. Cabrera, M. Marzo, J. M. Pares, and J. Agusti. 2001. A complete terrestrial Oligocene magnetobiostratigraphy from the Ebro Basin, Spain. Earth and Planetary Science Letters 187: 1-16.
Castresana, J. 2000. Selection of conserved blocks from multiple alignments for their use in phylogenetic analysis. Molecular Biology and Evolution 17:540-552.

Collar, D. C., J. A. Schulte II, B. C. O’Meara, and J. B. Losos. 2010. Habitat use affects morphological diversification in dragon lizards. Journal of Evolutionary Biology 23:1033-1049.

Covacevich, J., P. Couper, R. E. Molnar, G. Witten, and W. Young. 1990. Miocene dragons from Riversleigh: new data on the history of the family Agamidae (Reptilia: Squamata) in Australia. Memoirs of the Queensland Museum 29:339-360.

Daza, J. D., E. L. Stanley, P. Wagner, A. M. Bauer, and D. A. Grimaldi. 2016. Mid-Cretaceous amber fossils illuminate the past diversity of tropical lizards. Science Advances 2:e1501080.

Katoh, K., K. Misawa, K. Kuma, and T. Miyata. 2002. MAFFT: a novel method for rapid multiple sequence alignment based on fast Fourier transform. Nucleic Acids Research 30:3059-3066.

Kumar, S., G. Stecher, M. Suleski, and S. B. Hedges. 2017. TimeTree: a resource for timelines, timetrees and divergence times. Molecular Biology and Evolution 34: 1812-1819.

Lanfear, R., B. Calcott, S. Y. W. Ho, and S. Guindon. 2012. PartitionFinder: combined selection of partitioning schemes and substitution models for phylogenetic analyses. Molecular Biology and Evolution 29:1695-1701.

Martins, E. P., and T. F. Hansen. 1996. A microevolutionary link between phylogenies and comparative data. Pages 273-288 in P. H. Harvey, A. S. L. Brown, J. Maynard Smith, and S. Nee. New uses for new phylogenies. Oxford University Press, Oxford.

Paradis, E., J. Claude, and K. Strimmer. 2004. APE: analyses of phylogenetics and evolution in R language. Bioinformatics 20:289290.

Silvestro, D., A. Kostikova, G. Litsios, P. B. Pearman, and N. Salamin. 2015. Measurement errors should always be incorporated in phylogenetic comparative analysis. Methods in Ecology and Evolution 6:340-346.

Sovereign, O., C. Dullemeijer, P. van 't Veer, and H. van der Voet. 2012. Transformations of summary statistics as input in metaanalysis for linear dose-response models on a logarithmic scale: a methodology developed within EURRECA. BMC Medical Research Methodology 12:57.

Stadler, T. 2009. On incomplete sampling under birth-death models and connections to the sampling-based coalescent. Journal of Theoretical Biology 26:58-66.

Talavera, G., and J. Castresana. 2007. Improvement of phylogenies after removing divergent and ambiguously aligned blocks from protein sequence alignments. Systematic Biology 56:564577.

Associate Editor: Daniel L. Rabosky Editor: Daniel I. Bolnick 